\title{
SCALP: Scintillating ionization chamber for ALPha particle production in neutron induced reactions
}

\author{
B. Galhaut ${ }^{1,2, \text { a }}$, D. Durand ${ }^{2}$, F.R. Lecolley ${ }^{2}$, X. Ledoux $^{3}$, G. Lehaut ${ }^{2}$, L. Manduci ${ }^{1,2}$, and P. Mary ${ }^{1}$ \\ 1 Ecole des Applications Militaires de l'Energie Atomique, BP. 19, 50115 Cherbourg Armées, France \\ ${ }^{2}$ Laboratoire de Physique Corpusculaire de Caen, ENSICAEN/Normandie Univ./CNRS-IN2P3, 6 Boulevard Maréchal Juin, \\ 14050 Caen Cedex, France \\ ${ }^{3}$ Grand Accélérateur National d'Ions Lourds, CEA/DRF-CNRS/IN2P3, Boulevard Henri Becquerel, BP. 55027, 14076 Caen Cedex 5, \\ France
}

\begin{abstract}
The SCALP collaboration has the ambition to build a scintillating ionization chamber in order to study and measure the cross section of the $\alpha$-particle production in neutron induced reactions. More specifically on ${ }^{16} \mathrm{O}$ and ${ }^{19} \mathrm{~F}$ targets. Using the deposited energy (ionization) and the time of flight measurement (scintillation) with a great accuracy, all the nuclear reaction taking part on this project will be identify.
\end{abstract}

\section{Introduction and motivation}

In a nuclear reactor, alpha production due to neutron induced reactions on the oxygen of the uranium oxide is a sizeable contribution which affects the installation physical parameters. Oxygen is present in the water of thermal neutron nuclear reactor (as coolant and moderator) and also in fuel elements (uranium oxide or mixed oxide) of thermal and fast neutron reactor [1]. The ${ }^{16} \mathrm{O}(\mathrm{n}, \alpha)$ cross section reaction affects the $\mathrm{k}_{\mathrm{eff}}$ prediction (it is responsible for $100 \mathrm{pcm}$ on the total uncertainty). Moreover, $(\mathrm{n}, \alpha)$ reaction on light elements can be a significant source of helium production. The $(n, \alpha)$ reaction on oxygen contributes to an extent of $25 \%$ of the overall helium production in these kind of reactors. The uncertainty of helium production due to ${ }^{16} \mathrm{O}(\mathrm{n}, \alpha)$ is about $7 \%$.

Many evaluations were performed and great discrepancies were found among experimental measurements and also among the nuclear model predictions. Since 2005 efforts have been done by the Nuclear Energy Agency (NEA) in order to improve nuclear data. The cross section of the reaction $(n, \alpha)$ on oxygen figures in the compilation of the most important nuclear data requirements, i.e. in the High Priority Request List (HPRL). Its knowledge is required from threshold to $20 \mathrm{MeV}$ with a $5 \%$ accuracy in the whole range [2]. The necessity for measurements on this cross section was enhanced in [3,4] mostly for the nuclear reaction physics (especially about the reaction's influence on $\mathrm{k}_{\mathrm{eff}}$ underestimation). Since 2014 the CIELO Pilot Project [5] was devoted to improve the nuclear evaluations. The knowledge of the ${ }^{16} \mathrm{O}(\mathrm{n}, \alpha){ }^{13} \mathrm{C}$ cross section from the reaction threshold $(2.36 \mathrm{MeV})$ to at least $10 \mathrm{MeV}$ with a great accuracy is a major goal for the nuclear and the reactor physics.

\footnotetext{
a e-mail: galhaut@lpccaen.in2p3.fr; bastien.galhaut@ eamea.fr
}

\section{Experimental cross section measurements and evaluations}

Since 1955 several measurements have been performed over the ${ }^{16} \mathrm{O}(\mathrm{n}, \alpha)$ cross section for neutron energies below $10 \mathrm{MeV}$. It is possible to find in literature the works of Seitz and Huber [6], Davis et al. [7], Divatia et al. [8], Sekharan et al. [9], Dandy et al. [10], Giorginis et al. [11] and Khryachkov et al. [12,13]. The cross sections were estimated from the experimental values and nuclear model predictions. Some nuclear data library about nuclear reaction cross section evaluations exist. The last three most important libraries about the ${ }^{16} \mathrm{O}(\mathrm{n}, \alpha){ }^{13} \mathrm{C}$ reaction are ENDF/B-VII.1, JEFF-3.2 and JENDL-4.0.

Figures 1 and 2 show the ${ }^{16} \mathrm{O}(\mathrm{n}, \alpha)$ experimental cross section, up to $6 \mathrm{MeV}$ and from 6 to $8.5 \mathrm{MeV}$ respectively. They summarize all the experimental and evaluated values. Evaluations on ${ }^{16} \mathrm{O}(\mathrm{n}, \alpha){ }^{13} \mathrm{C}$ cross section are also represented. The main evaluations ENDF/BVII.1, are represented by the red line, JEFF-3.2 and JENDL-4.0 respectively by the dashed blue and the dashed black lines. In Fig. 2, the ENDF's evaluation on the partial cross section of the ${ }^{16} \mathrm{O}\left(\mathrm{n}, \alpha_{0}\right){ }^{13} \mathrm{C}$ reaction are represented by the dashed green line.

A close sight at Fig. 1 reveals the consistency of the different measurements and evaluations for neutrons energies below $6 \mathrm{MeV}$. All of them reproduce the resonances structures, even if the values found by Giorginis et al. [11] are systematically higher than the others evaluations and experimental data. Evaluations on the ${ }^{16} \mathrm{O}\left(\mathrm{n}, \alpha_{0}\right){ }^{13} \mathrm{C}$ cross section are also similar, JEFF32 and JENDL-4.0 are based on the former version of ENDF/B-VII.1 (ENDF/B-VII.0) below 6.5 MeV [5].

For energies above $6 \mathrm{MeV}$ (in Fig. 2) one can appreciate various discrepancies in the evaluated and experimental data, especially between 6 and $8 \mathrm{MeV}$. Data from Giorginis [11] and Khryachkov [12,13] are higher than the others data. In the range between 6 and 


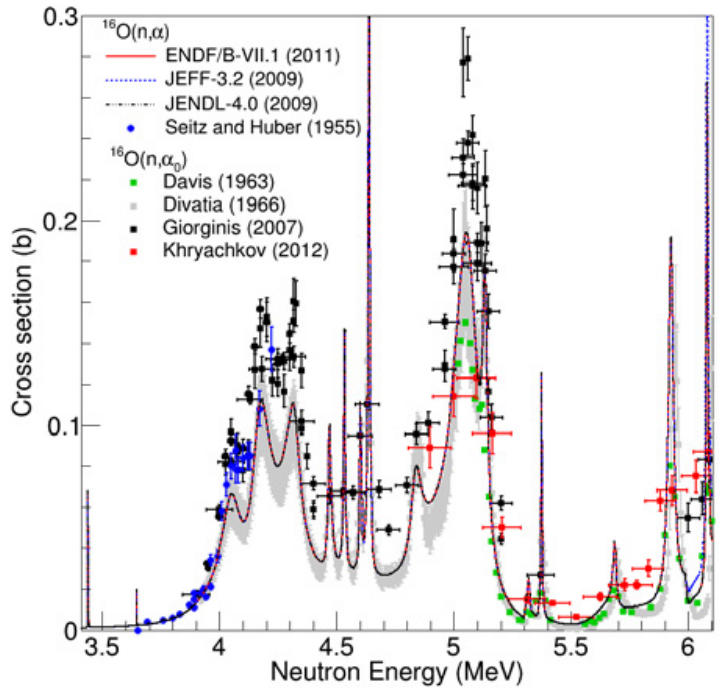

Figure 1. Significant cross section measurements and significant evaluations (from 3.5 to $6 \mathrm{MeV}$ ). Evaluations are represented in lines, cross section measurements in dots markers and partial cross section measurements in squared markers.

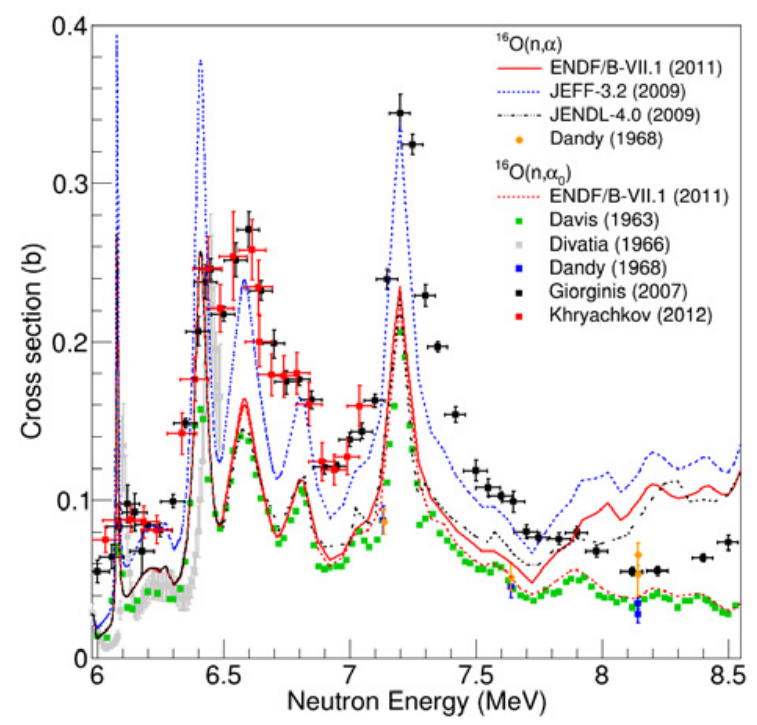

Figure 2. Significant cross section measurements and significant evaluations (from 6 to $8.5 \mathrm{MeV}$ ). Evaluations are represented in lines, cross section measurements in dots markers and partial cross section measurements in squared markers.

$8 \mathrm{MeV}$ also among the evaluations one can see important discrepancies. JEFF-3.2 evaluations on ${ }^{16} \mathrm{O}(\mathrm{n}, \alpha)$ are higher than the two others evaluations. ENDF/B-VII.1 evaluations on ${ }^{16} \mathrm{O}(\mathrm{n}, \alpha)$ and ${ }^{16} \mathrm{O}\left(\mathrm{n}, \alpha_{0}\right)$ reactions is based on experimental cross section data from Davis [7] and Dandy [10] among others data. The above discussion shows that the ${ }^{16} \mathrm{O}(\mathrm{n}, \alpha)$ cross section values show, at certain energies, uncertainties up to $30 \%$. This situation was considered unacceptable by the physicists involved in the reactor physics so that new projects of measurements, possibly with great accuracy, are demanded. Their goal is to reduce the uncertainties and the SCALP project belongs to this context.

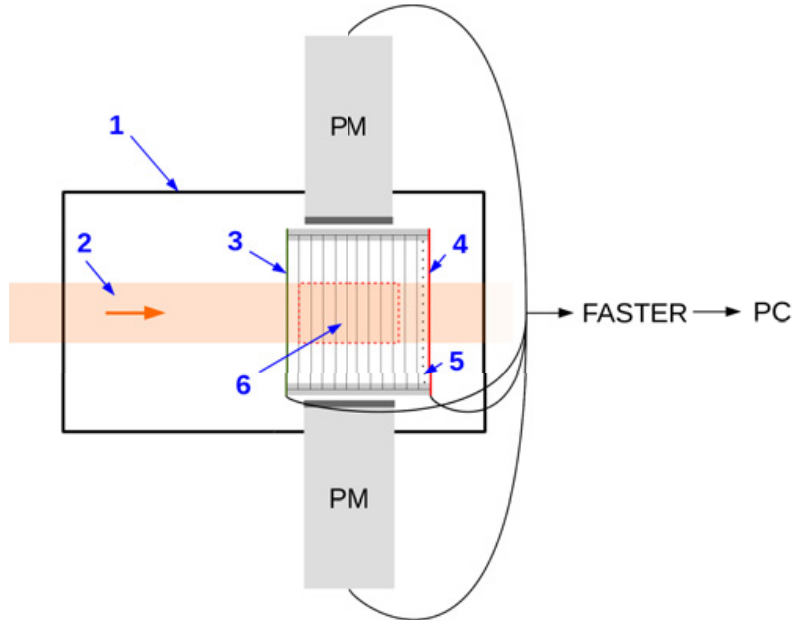

Figure 3. Schematic of SCALP's experimental setup. 1: external chamber. 2: Incident neutron flux. 3: Cathode (in green). 4: Anode (in red). 5: Frish grid. 6: Active volume (gas).

Table 1. Q-value and threshold of all the expected nuclear reactions (except elastic and inelastic nuclear reactions).

\begin{tabular}{c|c|c}
\hline Reaction & Q-value $(\mathrm{MeV})$ & Threshold $(\mathrm{MeV})$ \\
\hline${ }^{19} \mathrm{~F}(\mathrm{n}, \alpha)$ & -1.52 & 1.61 \\
${ }^{16} \mathrm{O}(\mathrm{n}, \alpha)$ & -2.22 & 2.36 \\
${ }^{19} \mathrm{~F}(\mathrm{n}, \mathrm{n}+\alpha)$ & -4.01 & 4.23 \\
${ }^{19} \mathrm{~F}(\mathrm{n}, \mathrm{p})$ & -4.04 & 4.25 \\
${ }^{12} \mathrm{C}(\mathrm{n}, \alpha)$ & -5.70 & 6.18 \\
${ }^{19} \mathrm{~F}(\mathrm{n}, \mathrm{d})$ & -5.77 & 6.08 \\
${ }^{16} \mathrm{O}(\mathrm{n}, \mathrm{n}+\alpha)$ & -7.16 & 7.61 \\
${ }^{19} \mathrm{~F}(\mathrm{n}, \mathrm{t})$ & -7.56 & 7.96 \\
${ }^{19} \mathrm{~F}(\mathrm{n}, \mathrm{n}+\mathrm{p})$ & -7.99 & 8.42 \\
\hline
\end{tabular}

\section{SCALP project}

In these conditions, the aim of the SCALP project is then to perform several measurements of the ${ }^{16} \mathrm{O}(\mathrm{n}, \alpha){ }^{13} \mathrm{C}$ cross section up to $10 \mathrm{MeV}$ and with an accuracy which will be improved in the progressive work. The experiment is based on the detection of the $\alpha$-particles produced in the reaction. The scintillating properties of $\mathrm{CF}_{4}$ [14] will allow the measurement of the energy of the incident neutron by time-of-flight measurements.

\subsection{Experimental setup}

SCALP is an ionization chamber filled with a mixture of $\mathrm{CF}_{4}$ and a few percent of $\mathrm{CO}_{2}$. Four photomultipliers tubes collect the light emission associated with the neutron induced reactions in the gas. In Fig. 3, the external chamber (1) is sealed and filled with a gas mixture (atmospheric pressure). The chamber is set on the incident neutron (2) path (from the Neutron For Science NFS facility). The inside ionization chamber dimensions $(100 \mathrm{~mm} \times$ $100 \mathrm{~mm} \times 130 \mathrm{~mm}$ ) and characteristics (see Fig. 3) were studied with the help of GEANT4 simulations (see Sect. 3.3). The gas active volume (6), in which the number of target atoms is known accurately, was also estimated by simulations. The ionization chamber is surrounded by the four photomultipliers tubes. A digital modular acquisition system FASTER [15] is connected next to the experimental setup. 


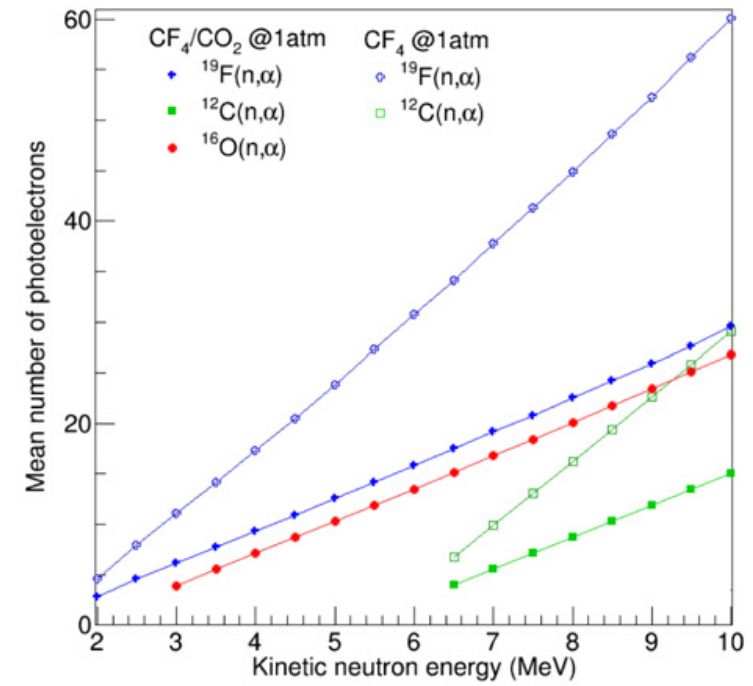

Figure 4. Estimation of mean number of photoelectrons of $(\mathrm{n}, \alpha)$ reactions (from GEANT4 simulations, using pure $\mathrm{CF}_{4}$ and $\mathrm{CF}_{4} / \mathrm{CO}_{2}$ at $\left.1 \mathrm{~atm}\right)$.

\subsection{Principle}

The use of a scintillating ionization chamber was selected in order to discriminate all the nuclear reactions involved in the time-of-flight measurements. The deposited energy by a charged-particle from the nuclear reaction is measured by ionization charges into the active gas volume between the two electrodes (cathode and anode) of the ionization chamber. Scintillation (from $\mathrm{CF}_{4}$ scintillating gas) allows to know the interaction time. The time-of-flight measurement is possible since the emission time of the neutrons from the NFS facility is known.

Table 1 lists the Q-values and thresholds of all the expected reactions (except elastic and inelastic reactions). The $(\mathrm{n}, \alpha)$ reactions from ${ }^{19} \mathrm{~F}$ and ${ }^{16} \mathrm{O}$ are separated by a difference in the Q-value of only $700 \mathrm{keV}$. The knowledge of both the deposited particle energy and the incident neutron energy (deduced from the neutron time-of-flight) is needed with a great accuracy. It is crucial to separate and identify all the nuclear reactions of interest. The accurate estimation of the nuclei-targets of fluorine and oxygen in the active volume will allow the knowledge of the cross section.

\subsection{GEANT4 simulations}

GEANT4 simulations helped to evaluate and to constraint the parameters of the scintillating ionization chamber. Varying the neutron energy, the evaluation of the light yield, the deposited energy, the time-of-flight neutron, the time-to-digital converter (TDC), and the position of the interaction was possible.

Figure 4 shows the expected light yield of $(n, \alpha)$ reactions in SCALP using a pure $\mathrm{CF}_{4}$ gas and a gas mixture of $\mathrm{CF}_{4}(97 \%)$ and $\mathrm{CO}_{2}(3 \%)$, both at a pressure of $1 \mathrm{~atm}$. In the mixture case the number of photoelectrons (from $5,6 \mathrm{MeV} \alpha$ particles) was reduced by two, in comparison to pure $\mathrm{CF}_{4}$. The use of the gas mixture allows a "great" number of oxygen nuclei-target while the light yield is limited. The time resolution of SCALP is deduced from the coincidence resolving time (CRT) between two photomultiplier tubes. Figure 5 shows the CRT versus the

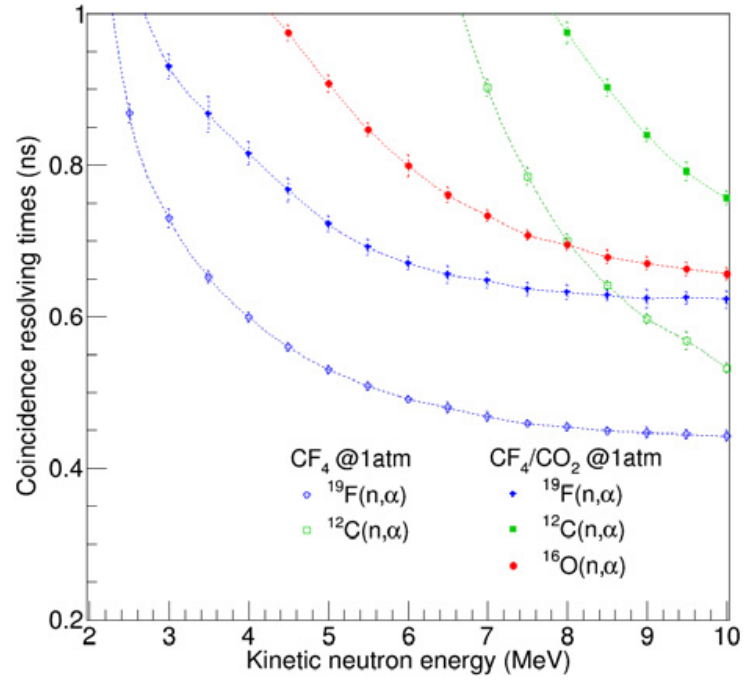

Figure 5. Estimation of $\mathrm{CRT}$ values of $(\mathrm{n}, \alpha)$ reactions (from GEANT4 simulations, using pure $\mathrm{CF}_{4}$ and $\mathrm{CF}_{4} / \mathrm{CO}_{2}$ at $1 \mathrm{~atm}$ ).

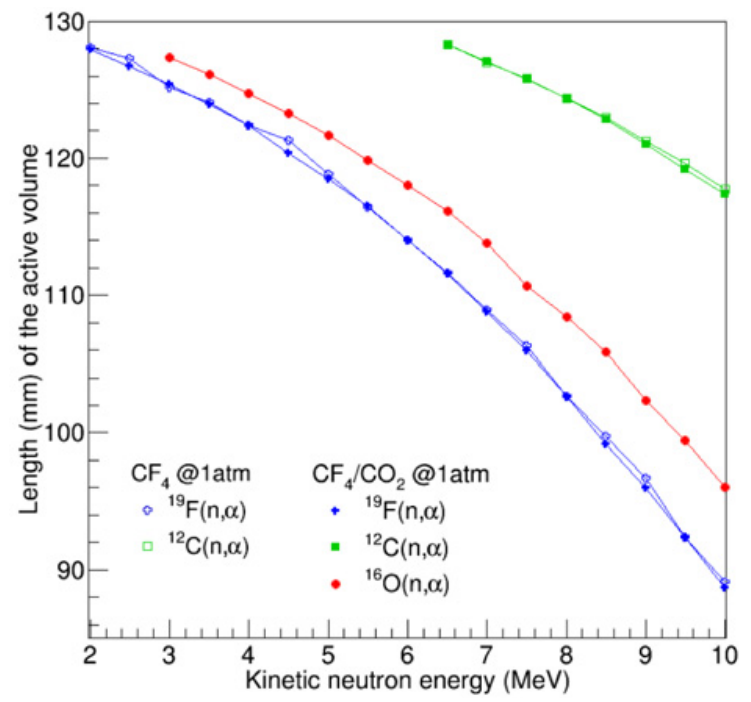

Figure 6. Estimation of active length of $(\mathrm{n}, \alpha)$ reactions (from GEANT4 simulations, using pure $\mathrm{CF}_{4}$ and $\mathrm{CF}_{4} / \mathrm{CO}_{2}$ at $1 \mathrm{~atm}$ ).

neutron energy. One can see that using the gas mixture the CRT increases of about a factor 1.44. The active volume was also estimated using GEANT4 simulations. The length of the active volume along the neutron path is shown in Fig. 6. This characteristics is important, since the cross section depends on the nuclei targets in the active volume. The length of the ionization chamber is $130 \mathrm{~mm}$. Up to $10 \mathrm{MeV}$ the active volume has a maximal length of $89 \mathrm{~mm}$.

Figure 7 shows the deposited energy versus the incident neutron time-of-flight. Signals from the ${ }^{19} \mathrm{~F}(\mathrm{n}, \alpha)$ reaction are represented in blue, the ones from ${ }^{16} \mathrm{O}(\mathrm{n}, \alpha)$ in red. As one can see, SCALP seems to be able to well discriminate between these two close reactions, at least up to $10 \mathrm{MeV}$ (more especially at around 95-100 ns incident neutron time-of-flight, which corresponds to roughly $13.5 \mathrm{MeV}$ ). This is promising for the cross sections measurements. 


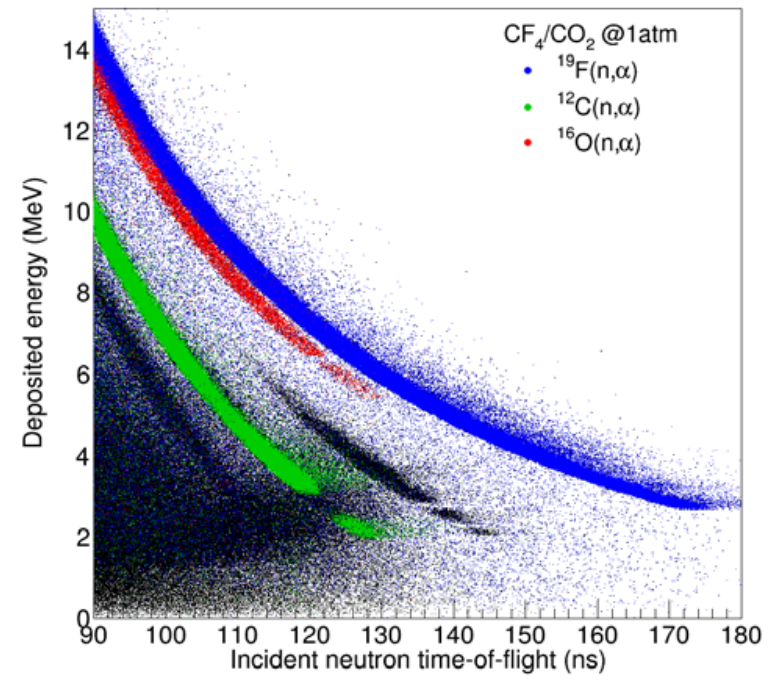

Figure 7. Discrimination of ${ }^{16} \mathrm{O}(\mathrm{n}, \alpha)$ (red), ${ }^{\text {nat }} \mathrm{C}(\mathrm{n}, \alpha)$ (green) and ${ }^{19} \mathrm{~F}(\mathrm{n}, \alpha)$ (blue) reactions (from GEANT4 simulations). The others nuclear reactions are in black.

\section{Conclusion}

As soon as the experimental setup is build, some laboratory tests will be carry out at LPC Caen and LPSC in Grenoble [16]. Then, cross section measurements using pure $\mathrm{CF}_{4}$ inside the SCALP setup will be performed at the Neutron For Science (NFS) facility at SPIRAL-2 [17]. The cross section of ${ }^{19} \mathrm{~F}(\mathrm{n}, \alpha){ }^{16} \mathrm{~N}$ will be measured. After analysis and feedback, the cross section of ${ }^{16} \mathrm{O}(\mathrm{n}, \alpha){ }^{13} \mathrm{C}$ will be estimated using the gas mixture $\left(\mathrm{CF}_{4} / \mathrm{CO}_{2}\right)$ at the NFS facility.

To conclude, these are some characteristics of the future SCALP device. The aim of this project is to measure $(n, \alpha)$ cross section on ${ }^{16} \mathrm{O}$ and ${ }^{19} \mathrm{~F}$. As we discussed above, SCALP will be able to distinguish these two reactions, using a gas mixture of $\mathrm{CF}_{4}(97 \%)$ and $\mathrm{CO}_{2}(3 \%)$. This will allow the $(\mathrm{n}, \alpha)$ cross sections measurements, at least up to $10 \mathrm{MeV}$ neutron energy.

\section{References}

[1] A. Courcelle, Working document "Need for ${ }^{16} \mathrm{O}(n, \alpha)$ Measurement and Evaluation in the Range 2.5 to $10 \mathrm{MeV}^{\prime \prime}(2005)$

[2] https://www.oecd-nea.org/dbdata/hprl/ hprlview $\cdot$ pl?ID $=417$

[3] A. Courcelle et al., Nuclear Data for Improved LEU-LWR Reactivity Predictions (NEA/WPEC-22, 2006) 41

[4] M. Salvatores et al., Uncertainty and Target Accuracy Assessment for Innovative Systems Using Recent Covariance Data Evaluations (NEA/WPEC26, 2008) 464

[5] M.B Chadwick and the CIELO collaboration, Nuclear Data Sheets 118, 1 (2014)

[6] J. Seitz and P. Huber, Helvetica Physica Acta 28, 227 (1955)

[7] E.A. Davis et al., Nuclear Physics 48, 169 (1963)

[8] A.S. Divatia et al., Proceedings of the Conference on Nuclear Data for Reactors (Paris) 1, 223 (1966)

[9] K.K. Sekharan et al., Physical Review 156, 1187 (1967)

[10] D. Dandy et al., A.W.R.E. Aldermaston Reports 60/68 (1968)

[11] G. Giorginis et al., Proceedings of International Conference on Nuclear Data for Science and Technology (Nice) 1, 525 (2007)

[12] V.A. Khryachkov et al., EPJ Web of Conferences 21, 03005 (2012)

[13] V.A. Khryachkov et al., Bulletin of the Russian Academy of Sciences: Physics 76, 486 (2012)

[14] G. Lehaut et al., Nuclear Instruments and Methods in Physics Research A 797, 57 (2015)

[15] http://faster.in2p3.fr/

[16] http://lpsc.in2p3.fr/

[17] X. Ledoux and the NFS collaboration, Nuclear Data Sheets 119, 353 (2014) 